\title{
The rise of a body through a rotating fluid in a container of finite length
}

\author{
By D. W MOORE† AND P. G. SAFFMAN \\ California Institute of Technology, Pasadena, California
}

(Received 19 April 1967 and in revised form 31 August 1967)

The drag on an axisymmetric body rising through a rotating fluid of small viscosity rotating about a vertical axis is calculated on the assumption that there is a Taylor column ahead of and behind the body, in which the geostrophic flow is determined by compatibility conditions on the Ekman boundary-layers on the body and the end surfaces. It is assumed that inertia effects may be neglected. Estimates are given of the conditions for which the theory should be valid.

\section{Physical discussion}

When a body moves slowly through a rotating liquid parallel to the axis of rotation it pushes ahead of it and pulls behind it a column of fluid in which there is no axial motion relative to the body. This effect was predicted by Taylor (1922), who confirmed it experimentally.

If the fluid is bounded axially this Taylor column will collide with these boundaries. Fluid in the Taylor column ahead of the body is pushed into a thin Ekman layer on the boundary, where it is deflected outwards (see figure 1 ). In a fluid of small viscosity, the decelerations of the axial flow in this Ekman layer and in a similar one on the body involve contraction of the vortex tubes of the rotation, so that negative relative axial vorticity appears upstream of the body. In the downstream Ekman layers there are accelerations, so that positive relative axial vorticity appears in the downstream column. The actual amount is given by the Ekman compatibility relation between the flux out of the Ekman layer and the relative vorticity outside it which shows that the relative axial vorticity is of order $U(\Omega / \nu)^{\frac{1}{2}}$, where $U$ is the axial speed of the body, $\Omega$ is the rotation rate and $\nu$ is the kinematic viscosity. There is thus a swirling motion with speed $O\left(R U \Omega^{\frac{1}{2}} / \nu^{\frac{1}{2}}\right)$ where $R$ is the body radius, opposing the rotation ahead of the body and a swirling motion of comparable order augmenting the rotation behind it. Thus in consequence of the geostrophic balance a smaller radial pressure gradient is required to contain the flow in the upstream column and a larger radial pressure gradient is required for containment downstream. Now the pressure outside the column is fixed so that the mean pressure upstream is increased and that

$\uparrow$ Permanent address : Department of Mathematics, Imperial College, London. 
downstream decreased by an amount $\rho_{0} R^{2} U \Omega^{\frac{3}{2}} / \nu^{\frac{1}{2}}$ where $\rho_{0}$ is the density of the liquid $\dagger$. Thus the body experiences a drag $D$ of order $\rho_{0} R^{4} U \Omega^{\frac{3}{2}} / \nu^{\frac{1}{2}}$. In dimensionless form, $D / \rho_{0} \Omega^{2} R^{4} \propto R o E^{-\frac{1}{2}}$, where $R o=U / \Omega R$ is the Rossby number and $E=\nu / \Omega R^{2}$ is the Ekman number. The object of the present note is to calculate a

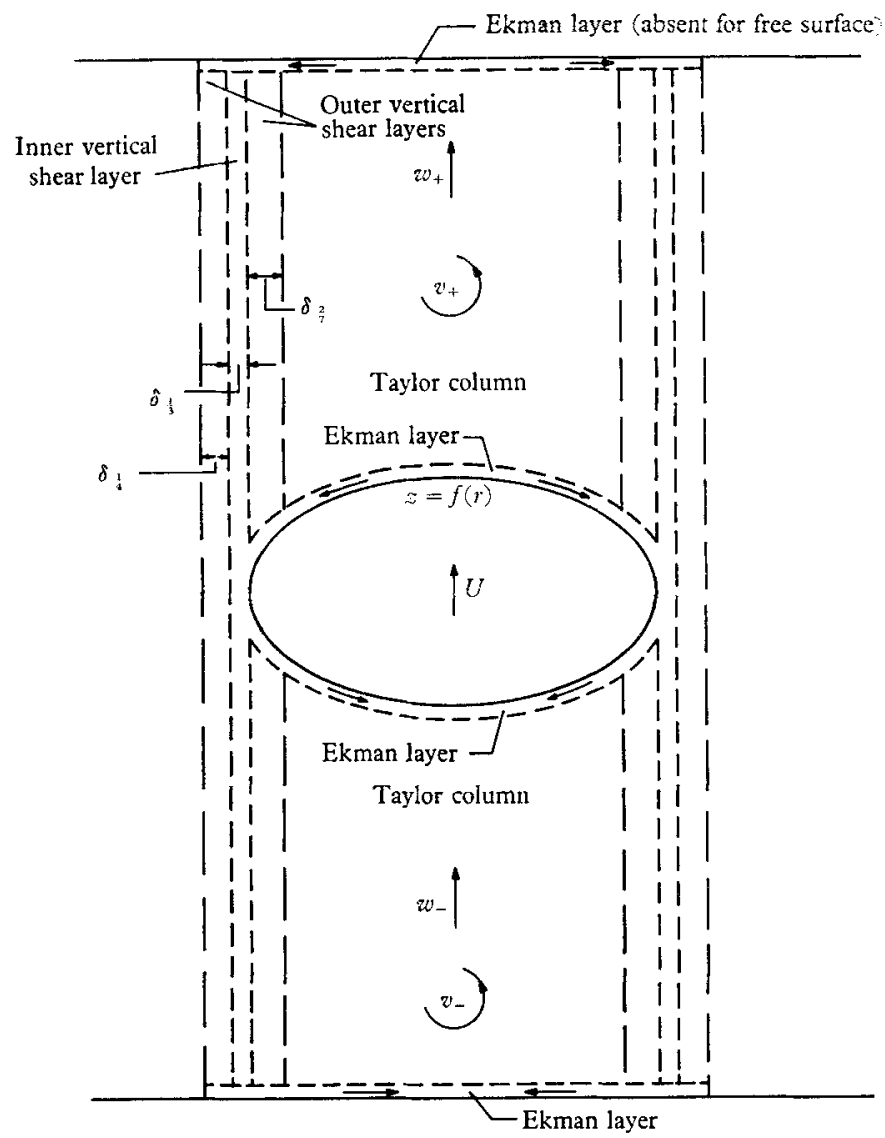

FIgURE 1. Sketch of motion produced by rising body.

precise value of this drag for axisymmetric bodies. It is assumed that the Ekman number is small enough so that the effects of viscosity are confined to the Ekman layers and to thin shear layers at the Taylor column boundary. Furthermore, it is assumed that the Rossby number is so small that the flow is geostrophically balanced outside the viscous regions. Then it is a straightforward matter to calculate the drag by going through a quantitative form of the above argument. This is done in $\S 2$ both for a container with rigid ends perpendicular to the rotation axis, and for a container rotating about a vertical axis whose upper surface is free. In this latter case there can be no Ekman layer at the free surface, so that there can be no axial motion outside the Ekman layer on the upper surface of the

$\dagger$ Pressure is measured relative to the pressure in uniform rigid body rotation. 
body. Thus this Ekman layer must be twice as intense to accomplish the deceleration, so that the pressure on the upper surface is approximately doubled. Thus there is about a $50 \%$ larger drag on the body when the container has a free surface. $\dagger$

In $\S 3$, we give estimates of the conditions for which the theory should be valid. The following paper (Maxworthy 1967) describes some experiments and compares the theory with observations.

\section{Derivation of the drag formulae}

We use cylindrical polar co-ordinates $(r, \theta, z)$ with $O z$ parallel to the axis of rotation. The velocity components are $(u, v, w)$. The shape of the side wall of the container does not affect the flow, but we assume that the base of the container is a rigid horizontal plane while the upper boundary is either a rigid horizontal plane also or a (slightly curved) free surface.

We shall consider only axisymmetric bodies and also suppose the body has fore and aft symmetry. The latter restriction is easily removed. The equation of the body is $z= \pm f(r), 0 \gtrless r \gtrless R$, relative to an origin moving with the body.

The quantity of interest is the drag $D$. If we use a subscript + to denote flow variables in the space $z>0$ and a subscript - to denote flow variable in $z<0$ we have that

$$
D=\int_{0}^{R} 2 \pi r\left\{p_{+}(r, f(r))-p_{-}(r,-f(r))\right\} d r
$$

the contribution from the viscous stress in the Ekman layer being negligible. Furthermore, since the change in pressure across the Ekman layer is also negligible for small Ekman number, we need to determine the pressure field only in the geostrophic interior. Here all the flow quantities are independent of $z$ and we have $-2 \rho_{0} \Omega v=-d p / d r$, which expresses the geostrophic balance. Thus our first step must be to determine the swirl velocity $v(r) . \S$

This is accomplished in a well known way by applying the Ekman compatibility relation to the Ekman layers on the container and boundaries and on the body. We suppose the body is rising with velocity $U$ and has angular velocity $\epsilon \Omega$ relative to the container.

Then for $0 \leqslant r<R$ we have the following relations between the axial velocity

$\dagger$ For a disk the drag is exactly $50 \%$ higher, since the Ekman layers on the disk and on the solid ends are identical.

¥ The drag on the body is balanced by the normal stresses over the container walls, the rate of change of vertical momentum of the fluid being smaller by a factor at most proportional to the Rossby number, so that the drag ean be evaluated by integrating the pressure over the flat end walls where the viscous normal stress is smaller by a factor of order $E$.

§ The exact velocity field is a function of time, since the body is moving relative to the walls, but for sufficiently small $U$ (the precise condition is $R o \ll E^{\frac{1}{2}}$ ) the flow field is quasisteady and the time dependence need not be considered explicitly. 
$w$ and the swirl velocity $v$ in the Taylor columns before and behind the body

$$
\left.\begin{array}{l}
w_{+}=-\frac{Q}{2 \lambda} \frac{1}{r} \frac{d}{d r}\left(r v_{+}\right) \quad \text { (upper surface of container), } \\
w_{+-}-U=\frac{1}{2 \lambda} \frac{1}{r} \frac{d}{d r}\left\{\left(r v_{+}-\epsilon r^{2} \Omega\right)\left(1+f^{\prime 2}\right)^{\frac{1}{4}}\right\} \quad \text { (upper surface of body), } \\
w_{-}-U=-\frac{1}{2 \lambda} \frac{1}{r} \frac{d}{d r}\left\{\left(r v_{-}-\epsilon r^{2} \Omega\right)\left(1+f^{\prime 2}\right)^{\frac{1}{4}}\right\} \quad \text { (lower surface of body), } \\
w_{-}=\frac{1}{2 \lambda} \frac{1}{r} \frac{d}{d r}\left(r v_{-}\right) \quad \text { (bottom wall of container), }
\end{array}\right\}
$$

where $Q$ is 0 for a free upper surface (since the free surface cannot support an Ekman layer) and is 1 for a rigid upper surface, and $\lambda=(\Omega / v)^{\frac{1}{2}}$. For $r>R$ we have only the first and last conditions which then show immediately that

$$
w=v=0 \text { for } r>R,
$$

so there is no relative motion outside the Taylor column. $\dagger$

For $0<r<R$ we find

and

$$
\begin{aligned}
& v_{+}=\frac{r\left[\epsilon \Omega\left(1+f^{\prime 2}\right)^{\frac{1}{4}}-U \lambda\right]}{Q+\left(1+f^{\prime 2}\right)^{\frac{1}{4}}} \\
& v_{-}=\frac{r\left[\epsilon \Omega\left(1+f^{\prime 2}\right)^{\frac{1}{4}}+U \lambda\right] .}{1+\left(1+f^{\prime 2}\right)^{\frac{1}{4}}}
\end{aligned}
$$

If we integrate the formula for the drag by parts we get

$$
D=\pi R^{2}\left\{p_{+}(R)-p_{-}(R)\right\}-\pi \int_{0}^{R} r^{2}\left\{\frac{d p_{+}}{d r}-\frac{d p_{-}}{d r}\right\} d r .
$$

Since the geostrophic balance equation holds for the radial pressure gradient even in the shear layer we have

$$
p(R+\delta)=p(R)+O\left(\rho_{0} \Omega v_{\delta} \delta\right),
$$

where $\delta$ is the shear layer thickness and $v_{\delta}$ is the magnitude of the swirl velocity in the shear layer. But $p$ is constant outside the shear layer, so

$$
p_{+}(R)-p_{-}(R)=O\left(\rho_{0} \Omega v_{\delta} \delta\right)
$$

It can be shown that $v_{\delta}$ is not greater than the swirl velocity in the Taylor column, so the pressure difference makes a negligible contribution to the drag. To calculate the integral we use the geostrophic balance equation and the above expressions for $v_{+}$and $v_{-}$. Thus we obtain

if the container has a rigid lid and

$$
D=4 \pi \rho_{0} \Omega \lambda U \int_{0}^{R} \frac{r^{3} d r}{1+\left(1+f^{\prime 2}\right)^{\frac{1}{4}}}
$$

$$
D=2 \pi \rho_{0} \Omega\left\{\lambda U \int_{0}^{R} r^{3} d r\left\{\frac{1}{\left(1+f^{\prime 2}\right)^{\frac{1}{4}}}+\frac{1}{1+\left(1+f^{\prime 2}\right)^{\frac{1}{4}}}\right\}-\epsilon \Omega \int_{0}^{R} \frac{r^{3} d r}{1+\left(1+f^{\prime 2}\right)^{\frac{1}{4}}}\right\}
$$

$\dagger$ The geostrophic equations allow the irrotational flow field $v=K / r$, but this velocity field requires Ekman layers which transport fluid to or from infinity and would therefore violate the conservation of mass. 
if the liquid has a free upper surface. The fact that the drag is independent of the spin in the former case is a consequence of the fore and aft symmetry.

Quantitative experiments on the motion of bodies in a contained rotating fluid have been done by releasing buoyant spheres at the bottom of the container (see following paper, Maxworthy 1967). Clearly a quasisteady state in which the buoyancy is balanced by the drag and in which the net torque is zero will be attained. Thus in order to compare our results for the drag we must determine $\epsilon \Omega$ by the condition that the torque is zero. Now in a steady state, the torque $T$ on the rising body is equal and opposite to the torque on the container ends. The $\theta$ component of stress on the lower plane boundary due to the Ekman layer is, from the familiar Ekman layer solution,

$$
\tau_{\theta}=\mu \lambda v_{-}
$$

so that, adding the contributions from the top and bottom surfaces,

$$
T=-\mu \lambda \int_{0}^{R} 2 \pi r^{2}\left(v_{-}+Q v_{+}\right) d r
$$

since the contributions from the regions outside the Taylor columns are negligible. Using the explicit formulae for $v$ we find that

$$
T=-4 \mu \lambda \pi \epsilon \Omega \int_{0}^{R} \frac{r^{3}\left(1+f^{\prime 2}\right)^{\frac{1}{4}} d r}{\left[1+\left(1+f^{\prime 2}\right)^{\frac{1}{1}}\right]}
$$

when both ends are rigid, and

$$
T=-2 \pi \mu \lambda\left\{U \lambda \int_{0}^{R} \frac{r^{3} d r}{\left[1+\left(1+f^{\prime 2}\right)^{\frac{1}{4}}\right]}+\epsilon \Omega \int_{0}^{R} \frac{r^{3}\left(1+f^{\prime 2}\right)^{\frac{1}{4}} d r}{\left[1+\left(1+f^{\prime 2}\right)^{\frac{1}{4}}\right]}\right\}
$$

when the upper surface is free.

In the symmetric case $T$ is independent of $U$ and vanishes when $\epsilon \Omega=0$, so that a freely rising body with fore-and-aft symmetry will rotate with the container. However, when the upper surface is free, the freely rising body will, since both integrals in the expression for the torque are positive, rotate more slowly than the container.

For a sphere of radius $R$ we find that for both ends rigid

$$
\frac{D}{\rho_{0}}=\frac{43}{105} \pi \Omega U \lambda R^{4}
$$

and that when the upper surface is free

$$
\frac{D}{\rho_{0}}=\frac{353}{630} \pi \Omega U \lambda R^{4}-\frac{43}{210} \pi \epsilon \Omega^{2} R^{4} .
$$

The torque vanishes in this case when

$$
\epsilon \Omega=-\frac{43}{62} U \lambda
$$

and the drag is then given by

$$
\frac{D}{\rho_{0}}=\frac{3919}{5580} \pi \Omega U \lambda R^{4}
$$

Note that the drag with a free upper surface is larger, as predicted in $\S 1$. 


\section{Discussion}

We will not discuss in detail the structure of the shear layer bounding the Taylor column, since the analysis is rather long and will be presented elsewhere. We will just mention one or two features which are important to the present study.

The shear layer for a blunt body has the sandwich structure discovered by Stewartson (1966) and consists of an inner layer of thickness $\delta_{\frac{1}{3}}=R E^{\frac{1}{3}}$, and two outer layers of thickness $\delta_{1}=R E^{\frac{1}{4}}$ and $\delta_{\frac{2}{7}}=R E^{\frac{2}{2}}$. The outer layers are associated with radial variations in swirl velocity. The inner layer is, however, unusual in having larger axial velocities than would be anticipated if (as has occasionally been asserted) its role were only to complete the circulation and to leading order the velocity field is a recirculating eddy. In fact, our analysis shows that in general the axial velocity in the inner layer is $O\left(U E^{-\frac{s}{18}}\right)$.

However, the case of a free body with fore-and-aft symmetry rising between plane rigid end walls is special, since it does not rotate relative to the fluid. Consequently, as (2.2) and (2.3) show, the geostrophic swirl velocity vanishes at the boundary of the Taylor column and the intensity of the shear layers is reduced. Our analysis shows that the recirculating axial velocity in the inner layer is now $O\left(U E^{-\frac{8}{2 i}}\right)$.

Our picture of the flow is given in figure 1. A necessary condition for the picture to hold is that the height of the container should be large compared with the Ekman layer thickness and the width of the outer shear layer should be small compared with $R$, and from a detailed analysis

$$
\left(\frac{\nu}{\Omega R^{2}}\right)^{\frac{1}{2}} \ll \frac{H}{R} \ll\left(\frac{\Omega R^{2}}{\nu}\right)^{\frac{1}{2}} .
$$

The second condition in (3.1) imposes an upper bound on the distance between the end walls. (As $H$ increases past this value, the flow pattern ceases to look like figure 1 until the walls are so far away that they cease to affect the flow. A geostrophic Taylor column then reappears, but it has a different structure and the drag is also an order of magnitude less. It can be shown that the end walls are unimportant if $H / R \gg E^{-1}$.)

A further condition for the calculation to be accurate is that inertia effects should be negligible. The relative order of magnitude of the inertia and Coriolis terms depends upon the region of flow. Thus inside the Taylor column and Ekman layers, the largest inertia term is the centrifugal force $\rho_{0} v^{2}$, which is small compared with the Coriolis force $\rho_{0} \Omega v$ if $\left(U^{2} / \Omega \nu\right) \ll 1$, on substituting the estimated magnitude of $v$. This condition can be written

$$
R o E^{-\frac{1}{2}} \ll 1 \text {, }
$$

in terms of the Rossby number and Ekman number.

The magnitude of the inertia effects in the shear layers are somewhat harder to estimate. However, investigation shows that inertia is generally negligible if it is small in the azimuthal momentum equation, and this requires $\partial v / \partial r \ll \Omega$. Putting in estimated magnitudes for the shear layer which result from a detailed analysis, we find

$$
R o E^{-\frac{3}{7}} \ll(H / R)^{\frac{3}{7}}
$$


for a free sphere rising with a rigid upper surface, and

if the upper surface is free.

$$
R o E^{-\frac{3}{4}} \ll(H / R)^{\frac{1}{2}}
$$

If we define critical Rossby numbers by (3.3) and (3.4) their ratio is $(H / R)^{\frac{1}{14}} E^{\frac{1}{28}}$, which is almost unity in any real situation. Inertia effects are probably more important when there is relative rotation, not because of any difference between (2.2) and (3.4), but because the velocity field has a different structure. Note that these conditions are more restrictive than (3.2) and the condition $R_{0} E^{-\frac{1}{3}} \ll 1$ given by Proudman (1956) for linearization in the flow between differentially rotating spherical surfaces. If (3.3) and (3.4) are not satisfied, the flow is unlikely to resemble that shown in figure 1 and the drag calculation will be in error.

Note that there is a Taylor column ahead of the body when the upper surface of the fluid is free, but it contains only an azimuthal velocity and not an axial velocity. If the surface of the body is also free, as would be the case for an air

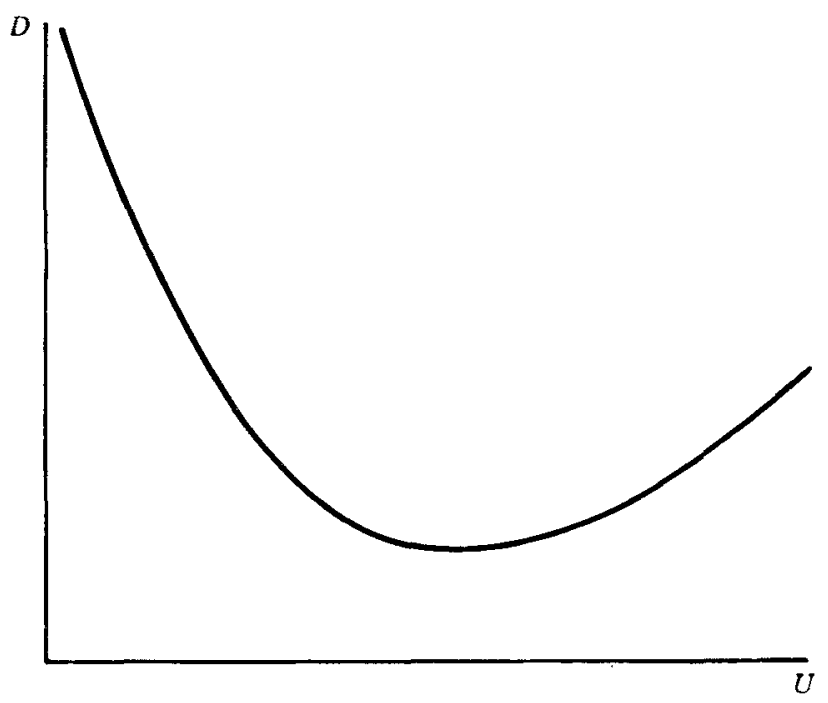

FIGURe 2. Drag of an air bubble as a function of its velocity of rise in a uniformly rotating fluid.

bubble, then there can be no Ekman layer on the upper surface of the body and there can be no flow out of the forward Taylor column. Thus if for an air bubble the flow configuration is of the type sketched in figure 1 , the Taylor column above the body has to be of constant length, which means that the bubble can not rise, or that the drag coefficient is infinite. $\uparrow$ This apparently paradoxical result raises the question whether the flow is of this type where the force on the body is given and not the velocity, as is the case for the experiments where a buoyant body is released in a rotating tank. There is the possibility that the body rises with a Rossby number greater than unity, in which case the drag is given to a first approximation by the theory for non-rotating fluids and may be smaller than the

$\dagger$ It is necessary to assume that the surface tension is sufficiently large to keep the bubble roughly spherical. 
drag at the much lower speeds required for the Taylor column to exist. For an air bubble, the curve of drag becomes infinite as the velocity and therefore the Rossby number tends to zero and increases with the velocity when rotation is unimportant (see figure 2). However, we are unable to estimate the position and value of the minimum.

For the case of a rigid body, the curve of drag against velocity is probably monotonic for the following reasons. For $R o \geqslant 1$, we have $D^{\prime} \sim R_{o}^{2}$ on the assumption that the drag coefficient is of order unity, where $D^{\prime}=D \rho \Omega^{2} R^{4}$. For (3.3) and (3.4) [which includes the case $R o E^{-\frac{1}{2}} \ll 1$ because of the restriction on $H / R]$, we have $D^{\prime} \sim R_{0} E^{-\frac{1}{2}}$. Thus $D^{\prime} \ll 1$ in the regime of validity of the low Rossby number flow, and there is no reason to expect a minimum in the transition region to high Rossby number.

There is of course always the possibility that the steady rectilinear motion may be unstable, particularly for a bubble, but this is a much harder problem. The stability of the shear layers is also an interesting question.

This research was partially supported by the Office of Naval Research under Contract Nonr 220(56).

\section{REFERENCES}

Maxworthy, T. 1967 The observed motion of a sphere through a short rotating cylinder of fluid. J. Fluid Mech. 31, 643.

Proudman, I. 1956 The almost-rigid rotation of viscous fluid between concentric spheres. J. Fluid Mech. 1, 505.

Stewartson, K. 1966 On almost rigid rotations. Part 2. J. Fluid Mech. 26, 131.

TAYLOR, G. I. 1922 The motion of a sphere in rotating liquid. Proc. Roy. Soc. A 102, 180. 